\title{
A high precision spectrum rectifying technique
}

\author{
J. Zhou ${ }^{1} \&$ Y. Zhong ${ }^{2,3}$ \\ ${ }^{1}$ School of Civil Engineering \& Architecture, \\ Chongqing Jiaotong University, China \\ ${ }^{2}$ School of Computer \& Information, Chongqing Jiaotong University, \\ China \\ ${ }^{3}$ Postdoctoral station, Chongqing University, China
}

\begin{abstract}
Spectrum-analysis technology has been widely used in engineering. Round discrete spectrum or FFT spectrum of a signal can be obtained rapidly by the FFT method. In practical applications, these FFT spectrums always need to be rectified in order to obtain a better spectrum. A high precision spectrum rectifying technique, a deconvolution-based spectrum rectifying technique, is developed in this paper. The sampled signal is firstly multiplied by an adaptive window function, the zooming spectrum of discrete windowed signal is then calculated, and finally the spectrum of window function is eliminated from the zooming spectrum by deconvolution so that the rectified spectrum of the analyzed signal can be obtained. Experiments show that this rectifying technique is very effective.
\end{abstract}

Keywords: spectrum rectifying technique, deconvolution, window function, sidepetal error.

\section{Introduction}

Frequency spectrum analysis technology has many applications in engineering [1]. FFT is frequently used to obtain signal spectrum rapidly. But the FFT spectrum with low resolution and sidepetal error caused by truncation is too rough to satisfy engineering demands. It induces the study on spectrum rectifying started from 1970 s of the $20^{\text {th }}$ century and many achievements have been made [2-4]. One of which is window function spectrum rectifying technique that decreases the sidepetal error by selecting a narrow-band window function. However, so far the rectified spectrum generated by this method is still 
mixed with the spectrum of window function. In this paper a deconvolutionbased spectrum rectifying technique is developed. It uses deconvolution technique adopted in recent years [5] to eliminate the spectrum of window function from the rectified spectrum so as to obtaining better rectified spectrum. Where the rectified spectrum is obtained by window function spectrum rectifying technique.

\section{Basics of the deconvolution-based spectrum rectifying technique}

Spectrum rectifying usually aims at two targets: improving frequency resolution and decreasing sidepetal error. In FFT, the frequency resolution $f_{1}$ of discrete spectrum is decided by the length $T$ of a truncated signal, i.e. $f_{1}=1 / T$. It seems that the frequency resolution won't be improved once $T$ has been determined. Nonetheless, only FFT algorithm restricts the frequency resolution to be $f_{1}=1 / T$. When sampling formula is satisfied, a discrete signal may represent a continuous signal. Therefore the spectrum of the discrete signal is also continuous and its frequency resolution may be arbitrary It is just the reason why the spectrum of discrete signal can be zoomed.

Following the literature [6], the continuous spectrum of the sampled signal $x\left(n T_{s}\right) \quad(n=-N / 2, \cdots,-1,0,1,2, \cdots, N / 2-1)$ can be calculated as

$$
\bar{X}(f)=T_{s} \sum_{n=-N / 2}^{N / 2-1} x(n) \exp (-j 2 \pi f n) .
$$

Therefore the zooming spectrum of signal with any time can be obtained from eqn. (1).

While when using eqn. (1), the result will be mixed sidepetal error since it truncates the analyzed signal, eg. adding a rectangular window on the analyzed signal. Let the analyzed signal be $x(t)$, the rectangular window function be $w(t)$, and the truncated signal be $\bar{x}(t)$, then we have

$$
\bar{x}(t)=x(t) w(t)
$$

and

$$
\bar{X}(f)=X(f) * W(f),
$$

where $X(f)$ is the spectrum of $x(t), \bar{X}(f)$ is the spectrum of $\bar{x}(t)$ and $\mathrm{W}(f)$ is the spectrum of $w(t)$.

According to eqn. (3), the spectrum $X(f)$ can be obtained by deconvolving to eliminate the spectrum $W(f)$ from $\bar{X}(f)$. 


\section{Algorithm of the deconvolution-based spectrum rectifying technique}

Since only discrete signal can be adopted in computer, let $\bar{X}(f)=X_{r}(f)+j \bar{X}_{i}(f), X(f)=X_{r}(f)+j X_{i}(f)$ and $W(f)=W_{r}(f)+j W_{i}(f)$, then we can obtain from eqn. (3)

$$
\bar{X}_{r}(f)=\int_{-\infty}^{\infty} X_{r}(u) W_{r}(f-u) d u-\int_{-\infty}^{\infty} X_{i}(u) W_{i}(f-u) d u
$$

and

$$
\bar{X}_{i}(f)=\int_{-\infty}^{\infty} X_{r}(u) W_{i}(f-u) d u+\int_{-\infty}^{\infty} X_{i}(u) W_{r}(f-u) d u .
$$

When frequency $f$ and $u$ are sampled with interval $f_{0}$, we obtain

$$
\bar{X}_{r}\left(k f_{0}\right) \approx \sum_{n=-\infty}^{\infty} X_{r}\left(n f_{0}\right) W_{r}\left(k f_{0}-n f_{0}\right) f_{0}-\sum_{n=-\infty}^{\infty} X_{i}\left(n f_{0}\right) W_{i}\left(k f_{0}-n f_{0}\right) f_{0}
$$

and

$$
\bar{X}_{i}\left(k f_{0}\right) \approx \sum_{n=-\infty}^{\infty} X_{r}\left(n f_{0}\right) W_{i}\left(k f_{0}-n f_{0}\right) f_{0}+\sum_{n=-\infty}^{\infty} X_{i}\left(n f_{0}\right) W_{r}\left(k f_{0}-n f_{0}\right) f_{0} .
$$

which can be simply denoted as

$$
\bar{X}_{r}(k) / f_{0} \approx \sum_{n=-\infty}^{\infty} X_{r}(n) W_{r}(k-n)-\sum_{n=-\infty}^{\infty} X_{i}(n) W_{i}(k-n)
$$

and

$$
\bar{X}_{i}(k) / f_{0} \approx \sum_{n=-\infty}^{\infty} X_{r}(n) W_{i}(k-n)+\sum_{n=-\infty}^{\infty} X_{i}(n) W_{r}(k-n) .
$$

When $n<-M / 2$ or $n \geq M / 2, X_{r}(n)=0, X_{i}(n)=0$, we have

$$
\begin{gathered}
\bar{X}_{r}(k) / f_{0} \approx \sum_{n=-M / 2}^{M / 2-1} X_{r}(n) W_{r}(k-n)-\sum_{n=-M / 2}^{M / 2-1} X_{i}(n) W_{i}(k-n) \\
(-M / 2 \leq k<M / 2)
\end{gathered}
$$

and

$$
\begin{gathered}
\bar{X}_{i}(k) / f_{0} \approx \sum_{n=-M / 2}^{M / 2-1} X_{r}(n) W_{i}(k-n)+\sum_{n=-M / 2}^{M / 2-1} X_{i}(n) W_{r}(k-n) . \\
(-M / 2 \leq k<M / 2)
\end{gathered}
$$

The equations can be expressed in matrix form as:

$$
\bar{X}_{r}^{M \times 1} / f_{0} \approx W_{r}^{M \times M} X_{r}^{M \times 1}-W_{i}^{M \times M} X_{i}^{M \times 1}
$$

and

$$
\bar{X}_{i}^{M \times 1} / f_{0} \approx W_{i}^{M \times M} X_{r}^{M \times 1}-W_{r}^{M \times M} X_{i}^{M \times 1}
$$

where $\bar{X}_{r}^{M \times 1}=\left\lfloor\bar{x}_{k}^{r}\right\rfloor, \bar{X}_{i}^{M \times 1}=\left\lfloor\bar{x}_{k}^{i}\right\rfloor, X_{r}^{M \times 1}=\left\lfloor x_{k}^{r}\right\rfloor, X_{i}^{M \times 1}=\left\lfloor x_{k}^{i}\right\rfloor, W_{r}^{M \times M}=\left\lfloor w_{k, n}^{r}\right\rfloor$, 


$$
\begin{aligned}
& W_{\mathrm{i}}^{M \times M}=\left[w_{k, n}^{i}\right], \quad \bar{x}_{k}^{r}=\bar{X}_{r}(-M / 2-1+k) / f_{0}, \quad \bar{x}_{k}^{i}=\bar{X}_{i}(-M / 2-1+k) / f_{0}, \\
& x_{k}^{r}=X_{r}(-M / 2-1+k), \quad x_{k}^{i}=X_{i}(-M / 2-1+k), \quad w_{k, n}^{r}=W_{r}(n-k), \\
& w_{k, n}^{i}=W_{i}(n-k) . k, n=-\mathrm{M} / 2,-\mathrm{M} / 2+1, \cdots,-1,0,1,2, \cdots, M / 2-1 .
\end{aligned}
$$

In fact, the rectangular window function $w(t)$ in eqn. (3) can be replaced by any window function $r(t)$. Let $R(f)=X_{r}(f)+j X_{i}(f)$ be the spectrum of $r(t)$, then eqn. (12) and eqn. (13) become

$$
\bar{X}_{r}^{M \times 1} / f_{0} \approx R_{r}^{M \times M} X_{r}^{M \times 1}-R_{i}^{M \times M} X_{i}^{M \times 1}
$$

and

$$
\bar{X}_{i}^{M \times 1} / f_{0} \approx R_{i}^{M \times M} X_{r}^{M \times 1}-R_{r}^{M \times M} X_{i}^{M \times 1},
$$

where $\quad \mathrm{R}_{r}^{M \times M}=\left[\mathrm{r}_{k, n}^{r}\right], \quad \mathrm{R}_{i}^{M \times M}=\left[\mathrm{r}_{k, n}^{i}\right], \quad \mathrm{r}_{k, n}^{r}=\mathrm{R}_{r}(n-k), \quad \mathrm{r}_{k, n}^{i}=\mathrm{R}_{i}(n-k)$,

$$
(k, n=-\mathrm{M} / 2,-\mathrm{M} / 2+1, \cdots,-1,0,1,2, \cdots, M / 2-1) \text {. }
$$

$X_{r}^{M \times 1}$ and $X_{i}^{M \times 1}$ can be obtained by eqn. (14) and eqn. (15) in computer, so $X\left(\mathrm{n} f_{0}\right)=X_{r}\left(\mathrm{n} f_{0}\right)+j X_{i}\left(\mathrm{n} f_{0}\right) \quad(-M / 2 \leq \mathrm{n}<M / 2)$, the rectifying spectrum of $x(t)$ with resolution $f_{0}$, can be obtained in computer.

If a proper window function is selected to make $\mathrm{R}_{i}^{M \times M} \equiv 0$, then eqn. (14) and eqn. (15) will be simplified as

$$
\bar{X}_{r}^{M \times 1} / f_{0} \approx R_{r}^{M \times M} X_{r}^{M \times 1}
$$

and

$$
\bar{X}_{i}^{M \times 1} / f_{0} \approx-R_{r}^{M \times M} X_{i}^{M \times 1} .
$$

So $X_{r}^{M \times 1}$ and $X_{i}^{M \times 1}$ can be expressed as

$$
X_{r}^{M \times 1} \approx\left(R_{r}^{M \times M}\right)^{-1} \bar{X}_{r}^{M \times 1} / f_{0}
$$

and

$$
X_{i}^{M \times 1} \approx-\left(R_{i}^{M \times M}\right)^{-1} \bar{X}_{i}^{M \times 1} / f_{0} .
$$

As a result, less calculation is needed to execute the algorithm.

Since both Gauss signal

$$
c(t)=e^{-(t / \tau)^{2}}
$$

and its spectrum

$$
C(f)=\sqrt{\pi} \tau e^{-(\pi f \tau)^{2}}
$$

are real and symmetric, and especially they both have a scale factor $\tau$ which make its width adjustable, Gauss signal is a proper window function to rectify FFT spectrum with deconvolution.

In the deconvolution-based spectrum rectifying technique, the purpose to process signal with a proper window function is to make the boundary of the 
analyzed signal trend to be zero so as to decreasing the sidepetal error. If the window function is too narrow, the analyzed signal will be truncated and the information of the analyzed signal will lost. Inversely, if the window function is too wide, the boundary of the one doesn't trend to be zero and undesirable sidepetal error will not be eliminated. In order to select window function effectively in deconvolution-based spectrum rectifying technique, a method to determine the scale factor $\tau$ of Gauss window function is adopted in this paper, i.e., set $\tau=T /(2 \sqrt{-\ln \varepsilon})$ if $c(t)$ is recognized as zero when $c(t) \leq \varepsilon$ and the analyzed time interval is $[-T / 2, T / 2]$.

In summary, if the sampled signal of the analyzed signal $x(t)$ is $x\left(n T_{s}\right)(n=-N / 2, \cdots,-1, \quad 0,1,2, \cdots, N / 2-1), T=N T_{s}$, and the zooming times is $p$ (which is relative to the frequency resolution $f_{1}=1 / T$ in FFT algorithm), the spectrum rectifying process with deconvolution will be as follows:

step1 Determine $T$ and $\varepsilon$, and calculate the scale of Gauss window function $\tau=T /(2 \sqrt{-\ln \varepsilon})$.

step2 Sample the Gauss signal $c(t)=e^{-(t / \tau)^{2}}$ in time period of $[-T / 2, T / 2]$ with sampling interval $T_{s}$ to obtain discrete Gauss signal $c\left(n T_{s}\right)(n=-N / 2, \cdots,-1,0,1,2, \cdots, N / 2-1)$.

step3 Obtain discrete windowed signal $\bar{x}\left(n T_{s}\right)=x\left(n T_{s}\right) c\left(n T_{s}\right)$, $(n=-N / 2, \cdots,-1,0,1,2, \cdots, N / 2-1)$.

step4 Use eqn. (1) to calculate the zooming spectrum of $\bar{x}\left(n T_{s}\right)$ with resolution $f_{0}=f_{1} / p=1 /(p T)$ and obtain $\bar{X}\left(k f_{0}\right)=\bar{X}_{r}\left(k f_{0}\right)+j \bar{X}_{i}\left(k f_{0}\right)$ $(k=-M / 2, \cdots,-1,0,1,2, \cdots, M / 2-1)$, where $M=p N$.

step5 Use eqn. (21) to calculate $R_{r}(k)=C\left(k f_{0}\right)(k=1-M, \cdots, M-1)$ and obtain matrix $R_{r}^{M \times M}$.

step6 Use eqn. (18) and eqn. (19) to obtain $X_{r}^{M \times 1}$ and $X_{i}^{M \times 1}$, and the rectified spectrum $X\left(k f_{0}\right)(k=-M / 2, \cdots,-1,0,1,2, \cdots, M / 2-1)$.

\section{Experiments}

\subsection{Simulation signal}

Let a simulation signal $x(t)=\sin (2 \pi \times 0.3 t)+\sin (2 \pi \times 0.5 t)$ be sampled with non-integral periodic interval $T_{s}=0.133 \mathrm{~s}$ and sampled length $N=100$, then a discrete signal with limited length $x\left(n T_{s}\right) \quad(-N / 2<n \leq N / 2-1)$ is obtained 
as shown in Figure 1(a). Its FFT amplitude spectrum is shown in Figure 1(b). A Gauss window function with $\tau=3.362$ is shown in Figure 1(c) and its amplitude spectrum is shown in Figure 1 (d). Processing signal $x(t)$ with the Gauss window function, a discrete windowed signal $\bar{x}\left(n T_{s}\right)(-N / 2<n \leq N / 2-1)$ of $x(t)$ is obtained as shown in Figure 1 (e). The relative FFT amplitude spectrum is shown in Figure 1 (f). A double zooming spectrum is obtained by using eqn. (1) to treat the signal $\bar{x}\left(n T_{s}\right)$ as shown in Figure $1(\mathrm{~g})$. Finally the rectifying result of the zooming spectrum with deconvolution eqn. (18) and eqn. (19) is shown in Figure 1 (h). The rectifying effect can be found obvious when comparing Figure 1(h) with Figure 1(b). Some noise in the spectrum shown in Figure 1 (h) is the error caused by the approximate calculation of eqn. (4) to eqn. (19).
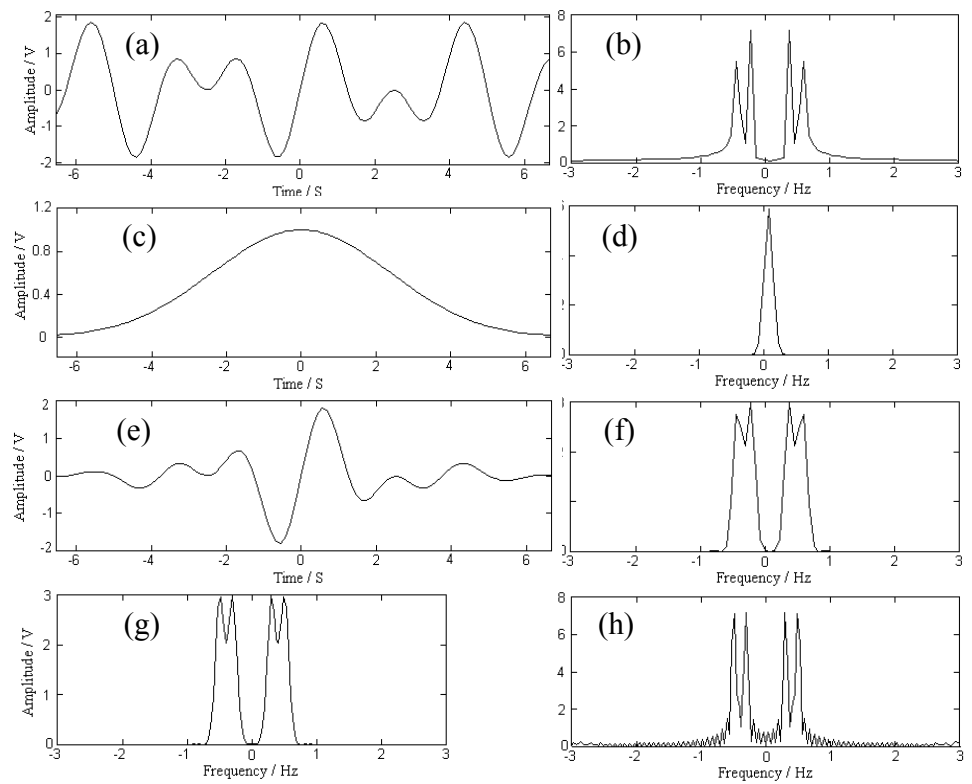

Figure 1: Simulation signal and its rectified spectrum.

\subsection{Random signal}

Figure2 (a) shows an airconditioner signal whose FFT amplitude spectrum is shown in Figure 2(b). The four-times zooming amplitude spectrum of windowed signal by Gauss window function is shown in Figure 2(c) and its rectified spectrum with deconvolution is shown in Figure 2(d). It is found that the sidepetal in FFT amplitude spectrum is eliminated effectively when comporing Figure 2(c) with Figure 2(d). 

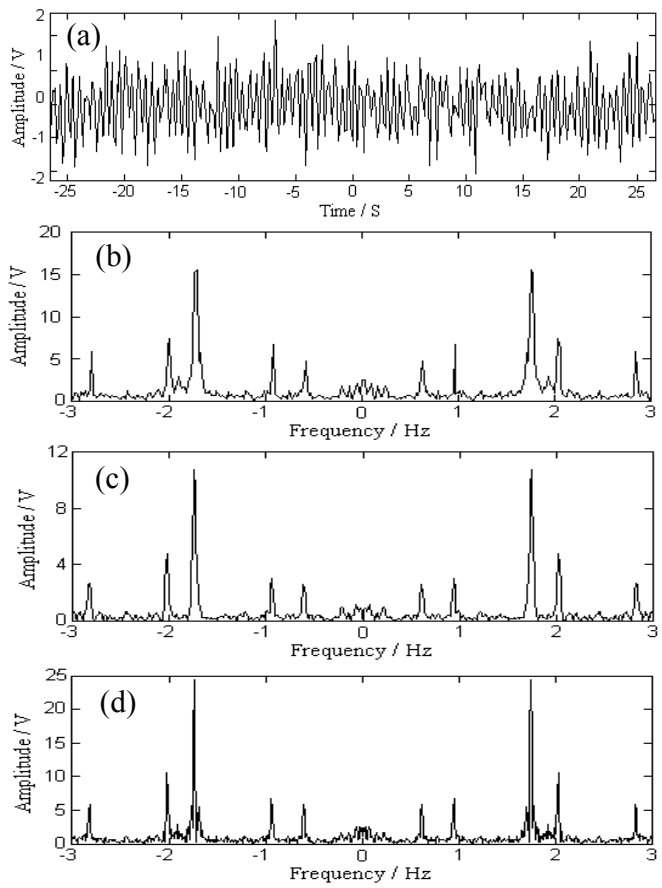

Figure 2: Air-condition signal and its rectified spectrum.

\section{Conclusions}

A deconvolution-based spectrum rectifying technique with the relative basement and algorithm is developed in this paper. Two notable points when using this technique are: (1) the window function must be properly selected, and (2) the scale of the window function must be adaptive to length $T$ of the analyzed signal. A simulation signal and a random signal experiment demonstrate that the rectifying technique is very effective.

\section{Acknowledgements}

The authors gratefully acknowledge the financial sponsorship from Major Technology Project of Chongqing City (No. CSTC2005AA6010), Western Communication Construction Science and Technology Project of Chinese Communication Ministry (No. 2004-318-814-26) and Natural Science Foundation of Chongqing City (No. CSTC, 2005BB6056).

\section{References}

[1] Hu, A.J., An, L.S. \& Tan, G.J., Application of spectrum correction theory in phase measurement for rotate machinery. Coal Mine Machinery, (10), pp.134-136, 2004. 
[2] Ding, K. \& Zhang, X.F., Advances in Spectrum Correction Theory. Journal of Vibration Engineering, 13(1), pp.14-22, 2000.

[3] Qian, K.M. \& Li, C.Q., New spectrum correction method based on chirp $\mathrm{Z}$ transform. Journal of Vibration Engineering, 13(4), pp.628-632, 2000.

[4] Li, T. Y. \& Ge, L.D., Research of two kinds of fast zoom spectrum. System Engineering and Electronics, 26(9), pp.1192-1194, 2004.

[5] Zhen, J.L., Yang, W.L. \& Ying, Q.H., Signal and Ayestem (the second version), Higher education press: Beijing, (the second volume), pp.35-36, 2000 .

[6] Zhong, Y.M., Qin, S.R. \& Tang, B.P. Some discussions on the extension principle in the DFT, Journal of Vibration and Shock, 20(4), pp.1-3, 2001. 